\title{
DISCRETE GROUPS AND NON-RIEMANNIAN HOMOGENEOUS SPACES
}

\author{
ROBERT J. ZIMMER
}

\section{INTRODUCTION}

A basic question in geometry is to understand compact locally homogeneous manifolds, i.e., those compact manifolds that can be locally modelled on a homogeneous space $J \backslash H$ of a finite-dimensional Lie group $H$. This means that there is an atlas on a manifold $M$ consisting of local diffeomorphisms with open sets in $J \backslash H$ where the transition functions between these open sets are given by translations by elements of $H$. A basic example is the case of $M=J \backslash H / \Gamma$ where $\Gamma \subset H$ is a discrete subgroup acting freely and properly discontinuously on $J / H$, with a compact quotient. (These examples can be abstractly characterized as the "complete" case. See [G].) We then call $\Gamma$ a cocompact lattice on $J \backslash H$. When $J$ is compact, $J \backslash H$ has an $H$-invariant Riemannian metric, so that $M$ itself becomes a Riemannian manifold in a natural way. Thus, the class of locally homogeneous Riemannian manifolds includes (but is not exhausted by) the locally symmetric spaces. For $H=$ $\operatorname{Aff}\left(\mathbb{R}^{n}\right)$ and $J=\operatorname{GL}(n, \mathbb{R})$, the spaces $J \backslash H / \Gamma$ are the complete affinely flat manifolds, a non-Riemannian example which has been extensively studied.

When $H$ is simple and $J$ is not compact, the situation is far from being well understood. Some special series of such homogeneous spaces admitting a cocompact lattice have been constructed by Kulkarni $[\mathrm{Ku}]$ and by $\mathrm{T}$. Kobayashi [K]. For example, Kulkarni shows the existence of such a discrete group $\Gamma$ for the homogeneous spaces $\mathrm{SO}(1,2 n) \backslash \mathrm{SO}(2,2 n)$ and Kobayshi does the same for $\mathrm{U}(1, n) \backslash \mathrm{SO}(2,2 n)$. On the other hand, the constructions they give for these (and other similar) series are quite special, and there has been the general suspicion that most homogeneous spaces $J \backslash H$ with $H$ simple and $J$ not compact do not admit a cocompact lattice. (We recall that, when $J$ is compact, i.e. the Riemannian case, such $\Gamma$ always exist by a result of Borel [B].) One general situation in which it is known that no such $\Gamma$ exists is the case in which $J \backslash H$ is noncompact and of reductive type and $\mathbb{R}-\operatorname{rank}(H)=\mathbb{R}-\operatorname{rank}(J)$. This situation was studied by Calabi-Markus [CM], Wolf [W], and Kobayashi [K], and the nonexistence of $\Gamma$ in this situation is a special case of a more general result known as the Calabi-Markus phenomenon. Kobayashi and Ono [KO] use the Hirzebruch proportionality principle to show that some other special series do

Received by the editors September 28, 1992.

1991 Mathematics Subject Classification. Primary 20H15, 22E40, 53C15, 53C30.

Research partially supported by NSF grant DMS 9107285. 
not admit cocompact lattices. Recently Benoist and Labourie [BL] have shown that if $H$ is simple and the center $Z(J)$ contains a nontrivial $\mathbb{R}$-split oneparameter subgroup then no such $\Gamma$ exists. Their result in fact applies more generally to show that there is no compact $M$ locally modelled on $J \backslash H$.

These results are quite special of course and, in particular, give no insight into the question whether such $\Gamma$ exist for the basic test case of $\operatorname{SL}(p, \mathbb{R}) \backslash \operatorname{SL}(n, \mathbb{R})$ where $p<n$ and the embedding is the standard one. The goal of this paper is to prove a (still special) result that clarifies the situation for the above examples in case $p<n / 2$ and for many other natual examples as well. Here is the main result.

Theorem 1.1. Let $H$ be a real algebraic group and $J \subset H$ an algebraic subgroup. Suppose:

(i) $H$ and $J$ are both unimodular;

(ii) There is a discrete group $\Gamma \subset H$ that is a cocompact lattice for $J \backslash H$; and

(iii) The centralizer $Z_{H}(J)$ of $J$ in $H$ contains a subgroup $G$ with the following properties:

(a) $G$ is not contained in a proper normal subgroup of $H$,

(b) $G$ is a semisimple Lie group each of whose simple factors has $\mathbb{R}$-rank at least 2 ,

(c) every local homomorphism of $G$ to $J$ is trivial.

Then $J$ is compact.

Corollary 1.2. Let $H$ be an almost simple real algebraic group. Suppose $J \subset H$ is a unimodular subgroup such that $Z_{H}(J)$ contains a simple Lie group of $\mathbb{R}$ rank at least 2 that does not have a local embedding in $J$. If $J \backslash H$ admits a cocompact lattice, then $J$ is compact.

Corollary 1.3. If $2 \leq p<n / 2, n>4$, then $\operatorname{SL}(p, \mathbb{R}) \backslash \operatorname{SL}(n, \mathbb{R})$ does not admit a cocompact lattice. The same is true for $J \backslash \operatorname{SL}(n, \mathbb{R})$ where $J$ is any unimodular noncompact subgroup of $\operatorname{SL}(p, \mathbb{R})$.

The remainder of the paper is devoted largely to the proof of Theorem 1.1. The proof involves in a crucial way results and arguments of algebraic ergodic theory, using in particular superrigidity for actions on principal bundles [Z1] and Ratner's theorem showing the validity of Raghunathan's measure conjecture [R]. Some parts of the argument are closely related to the arguments of [Z5].

We conclude this introductory section with some remarks and questions.

Questions. (1) With $J \backslash H$ as in Theorem 1.1 and $J$ noncompact, do there exist compact manifolds locally modelled on $J \backslash H$ ? While some of the ideas in the proof of Theorem 1.1 can be applied in this more general situation, more work is required to resolve this general case.

(2) Consider for simplicity the case of $H$ simple and $J \subset H$ unimodular. It is natural to ask if Corollary 1.2 remains true under weaker hypotheses on $Z_{H}(J)$. The proof of Theorem 1.1 and the results of [CZ] show that the conclusion of Corollary 1.2 still remains true if we only assume $Z_{H}(J)$ contains a noncompact simple group with Kazhdan's property, e.g., $\operatorname{Sp}(1, n)$. On the other hand, there are no known examples of $J \backslash H$ admitting a compact lattice where $H$ is simple, $J$ is noncompact and unimodular, and $Z_{H}(J)$ contains 
$\mathrm{GL}(2, \mathbb{R})$. It would, of course, be of interest to know if the conclusion of Corollary 1.2 remains true in this case as well.

(3) Are there cocompact lattices for $\operatorname{SL}(n-2, \mathbb{R}) \backslash \operatorname{SL}(n, \mathbb{R})$ or $\operatorname{SL}(n-1, \mathbb{R}) \backslash \operatorname{SL}(n, \mathbb{R})$ ? Even the case $\operatorname{SL}(2, \mathbb{R}) \backslash \operatorname{SL}(3, \mathbb{R})$ remains open.

The author's interest in the general issues this paper deals with was stimulated by the paper of Benoist-Labourie and many conversations with Labourie. We would like to thank Y. Benoist, P. Foulon, and F. Labourie for some very useful conversations that helped clarify some important issues involved in the main result. Special thanks are also due to S. G. Dani with whom I spent many productive hours discussing the arguments of the proof, and to F. Labourie for pointing out a gap in the original version of this paper. Much of the work on this paper was completed at the Institut des Hautes Études Scientifiques which we thank for its hospitality.

\section{Preliminaries}

In this section we provide one simple background result we shall need and briefly recall some standard notions we shall use freely.

Proposition 2.1. Let $H$ be a unimodular Lie group, $J \subset H$ a closed unimodular subroup, and $\Gamma \subset H$ a discrete subgroup that is a cocompact lattice for $J \backslash H$. Then there is a volume form on the compact manifold $M=J \backslash H / \Gamma$ that is invariant under the natural left action of $Z_{H}(J)$ on $M$.

Proof. Since both $H$ and $J$ are unimodular, there is an $H$-invariant volume form $\omega$ on $J \backslash H$ that is unique up to a constant multiple. If $g \in Z_{H}(J)$, then $g$ acts on $J \backslash H$ on the left commuting with the right action of $H$. Since $g^{*} \omega$ is then also $H$-invariant, it must be a scalar multiple of $\omega$, say $g^{*} \omega=c(g) \omega$. Since $\omega$ is $\Gamma$-invariant, it factors to a volume form $\omega_{0}$, and on $M$ we then clearly also have $g^{*} \omega_{0}=c(g) \omega_{0}$. Since $M$ is compact, $\omega_{0}$ defines a finite measure $\mu$ on $M$ and $g_{*} \mu=c(g) \mu$. Since the total measure is preserved, $c(g)=1$, and $\omega_{0}$ is $Z_{H}(J)$-invariant.

We shall deal extensively with actions of a Lie group by automorphisms of a principal bundle. We recall some standard notation regarding this situation.

Let $M$ be a manifold and $P \rightarrow M$ a principal $H$-bundle where $H$ is a Lie group, and suppose $G$ acts on $P$ by principal bundle automorphisms. If $V$ is any left $H$-space, we can form the associated bundle $E_{V}=(P \times V) / H \rightarrow P / H=$ $M$ with $V$ as fiber, and $G$ acts by bundle automorphisms of $E_{V} \rightarrow M$. Any measurable section $s$ of $P \rightarrow M$ (which always exists) defines a measurable trivialization $P \cong M \times H \rightarrow M$. The $G$ action on $M \times H$ corresponding to the action on $P$ under this trivialization will be given by $g \cdot(m, h)=$ $(g m, \alpha(g, m) h)$ where $\alpha: G \times M \rightarrow H$ is a measurable cocycle. We recall that two cocycles $\alpha, \beta: G \times M \rightarrow H$ are equivalent if there is a measurable $\varphi$ : $M \rightarrow H$ such that $\varphi(g m)^{-1} \alpha(g, m) \varphi(m)=\beta(g, m)$. Different trivializations of $P$ yield cocycles equivalent to $\alpha$, and, conversely, any cocycle equivalent to $\alpha$ comes from a trvialization. There are a large number of results concerning the structure of measurable cocycles or, equivalently, the measurable structure of actions on principal bundles. In particular, the superrigidity theorem for 
cocycles $[\mathrm{Z1}-\mathrm{Z} 3]$ is such a result, and there are several such results related to amenability and Kazhdan's property [Z7]. The notion of algebraic hull of a cocycle (or of an action on a principal bundle) is a basic invariant. We refer the reader to [Z1, Z4, Z9] for background information on all these and related issues.

\section{Proof of MaIn Theorem}

Let $H$ be a connected simple Lie group with finite center and $J \subset H$ an algebraic unimodular subgroup. We can assume $J$ is connected. Let $\Gamma \subset H$ be a discrete group, which we assume acts freely and properly discontinuously on $J \backslash H$ such that $M=J \backslash H / \Gamma$ is compact. We assume $Z_{H}(J)$ contains a simple Lie group of real rank at least 2 . We write the Lie algebra $\mathfrak{z}_{\mathfrak{h}}(\mathfrak{j})=\mathfrak{l} \ltimes \mathfrak{r}$ where $\mathfrak{r}$ is the radical and $\mathfrak{l}$ is a semisimple Levi factor. We let $G \subset Z_{H}(J)$ be the connected semisimple group corresponding to the sum of all simple factors of $\mathfrak{l}$ of real rank at least 2 . Then $G$ is a semisimple Lie group each of whose simple factors has $\mathbb{R}$ rank of at least 2 , and if $\sigma: G \rightarrow Z_{H}(J)$ is any faithful representation then $\sigma(G)$ and $G$ are conjugate in $Z_{H}(J)$.

We view $J \backslash H \rightarrow M$ as a principal $\Gamma$-bundle on which $G$ acts by $\Gamma$-bundle automorphisms. We have a natural embedding $\Gamma \rightarrow H$, and we let $Q \rightarrow M$ be the associated $H$-bundle, i.e., $Q=(J \backslash H \times H) / \Gamma$. The group $G$ then acts on $Q$ by $H$-bundle automorphisms, and we wish to apply superrigididy for actions on principal bundles. To this end, we let $(E, \nu)$ be the space of ergodic components of the $G$-action on $M$. Thus, $(E, \nu)$ is a measure space and there is a $G$-invariant measurable map $p: M \rightarrow E$ such that, letting $\mu$ be the measure on $M$, we have $\mu=\int_{e \in E}^{\oplus} \mu_{e} d \nu(e)$, where $\mu_{e}$ is a measure on $M_{e}=p^{-1}(e)$. Furthermore, a.e. $\mu_{e}$ is a $G$-invariant ergodic probability measure. For each $e$, we let $Q_{e} \rightarrow M_{e}$ the (measurable) principal $H$-bundle obtained by restricting $Q$ to the fibers over $M_{e}$. Since the action of $G$ on $M_{e}$ is ergodic, there is a well-defined algebraic hull for the action of $G$. We recall that there this is the unique conjugacy class of algebraic subgroups of $H$ such that there is a measurable $G$-invariant reduction of the bundle to an element of this conjugacy class but not to any smaller algebraic subgroup. We shall use basic properties of algebraic hulls freely and refer the reader to [Z4] for discussion of this notion.

We shall also find the following notation (from [Z9]) convenient.

Definition 3.1. If $P \rightarrow M$ is a principal $\Gamma$-bundle with $\Gamma$ discrete, $\Lambda \subset \Gamma$ is a subgroup, and $G$ acts by automorphisms, we say that the $G$-action is $\Lambda$ reducible if there is a measurable $G$-invariant reduction of $P$ to $\Lambda$.

We then have the following easy fact. (Cf. [Z9].)

Lemma 3.2. Suppose $P \rightarrow M$ is a principal $\Gamma$-bundle and that $G$ acts ergodically on $M$. Suppose $\Gamma \rightarrow H$ is a discrete faithful representation into an algebraic group $H$. Let $Q$ be the associated $H$-bundle. If the algebraic hull for the $G$ action on $P$ is compact, then $P \rightarrow M$ is $\Lambda$-reducible for some finite $\Lambda \subset \Gamma$.

(The proof of Lemma 3.2 also follows from the proof of Proposition 3.6 below.) 
Proposition 3.3. For almost all $e \in E$, the algebraic hull of the action of $G$ on $Q_{e} \rightarrow M_{e}$ is noncompact.

Proof. If this were not the case, then there would be a set of $e \in E$ positive measure for which the algebraic hull is compact. By Lemma 3.2, on a set $e \in E$ of positive measure, the $G$ action on $(J \backslash H)_{e} \rightarrow M_{e}$ is $\Lambda_{e}$-reducible for some finite $\Lambda_{e} \subset \Gamma$. It follows that each such $(J \backslash H)_{e}$ supports a finite $G$-invariant measure projecting to $M_{e}$, and hence (by integrating) $J \backslash H$ supports a finite $G$-invariant measure projecting to the restriction of $\mu$ to a subset of positive $\mu$-measure in $M$. Since the fibers of $J \backslash H \rightarrow M$ are discrete, it follows that $J \backslash H$ admits a finite $G$-invariant measure that is the restriction of a smooth measure to a $G$-invariant set. Since the action of $G$ on $J \backslash H$ is an action on a variety, the Borel density theorem implies that finite invariant measures are supported on fixed points. Therefore, we would have a set of fixed points of positive Lebesgue measure. Since the action is algebraic, this implies that $G$ acts trivially on $J \backslash H$, which is clearly impossible because $G$ is not contained in a proper normal subgroup of $H$.

Remarks. (a) Exactly the same argument can be applied to the action of any simple normal subgroup of $G$.

(b) In general, by passing to a finite ergodic extension, the algebraic hull will become a subgroup of finite index. Thus, Proposition 3.3 remains true if $Q_{e} \rightarrow M_{e}$ is replaced by the pullback of $Q_{e}$ to a finite ergodic extension of $M_{e}$.

We now fix an ergodic component $e$ that satisfies the conclusions of Proposition 3.3 and apply superrigidity for cocycles (i.e., for actions on principal bundles) in the form of $[Z 1, Z 3]$. We obtain:

Theorem 3.4. Fix $e$ as above. Then there is a finite ergodic extension $M_{e}^{\prime} \rightarrow M_{e}$ of $M_{e}$, a locally faithful representation $\sigma: \widetilde{G} \rightarrow H$, a compact subgroup $K \subset H$ with $K \subset Z_{H}(\sigma(\widetilde{G}))$ and a measurable function $\psi: M_{e}^{\prime} \rightarrow H$ such that

$$
\psi(g m)^{-1} \alpha^{\prime}(g, m) \psi(m)=\sigma(g) c(g, m)
$$

for $g \in \widetilde{G}, m \in M_{e}^{\prime}$ where $\alpha: G \times M_{e} \rightarrow \Gamma$ is the cocycle corresponding to $(J \backslash H)_{e} \rightarrow M_{e}, \alpha^{\prime}$ is its lift to $\widetilde{G} \times M_{e}^{\prime}$, and $c(g, m) \in K$.

We remark that $\sigma$ is nontrivial by Proposition 3.3 (and Remark (b)) and, in fact, is locally faithful by Remark (a).

By passing to a further ergodic extension with finite invariant measure, we can obtain the same conclusion but with c trivial. Namely, let $K_{0} \subset K$ be the algebraic hull of $\mathrm{c}$. Then $\mathrm{c}$ is equivalent to a cocycle $c_{0}$ taking values in $K_{0}$, and we can form the $G$-space $X_{e}=M_{e}^{\prime} \times c_{0} K_{0}$. Since $K$ is compact, this will be ergodic [Z6] (with respct to the finite $G$-invariant product measure). The pullback of $c_{0}$ to a cocycle $\widetilde{G} \times X_{e} \rightarrow K_{0}$ will be trivial. Therefore, we deduce

Corollary 3.5. There is a measurable function $\varphi: X_{e} \rightarrow H$ such that

$$
\varphi(g x)^{-1} \alpha_{X}(g, m) \varphi(g x)=\sigma(g)
$$


where $\alpha_{X}$ is the lift of $\alpha$ to a cocycle $\widetilde{G} \times X \rightarrow \Gamma$.

Now let $\lambda: X_{e} \rightarrow \Gamma \backslash H$ be the composition of $\varphi$ with the projection of $H$ to $\Gamma \backslash H$. Since $\alpha_{X}$ takes values in $\Gamma$, we deduce $\lambda(g x)=\lambda(x) \cdot \sigma(g)^{-1}$, i.e., that $\lambda$ is a $\widetilde{G}$ map. The measure $\lambda_{*}\left(\mu_{e}\right)$ is then a finite ergodic $\sigma(\widetilde{G})$ invariant measure on $\Gamma \backslash H$. As in [Z5] we can apply Ratner's theorem [R] to this situation.

Proposition 3.6. There is a connected Lie group $L \subset H$ such that:

(i) $\Lambda=L \cap \Gamma$ is a lattice in $L$, and

(ii) the $G$ action on the principal $\Gamma$-bundle $P_{X} \rightarrow X$, the pullback of $(J \backslash H)_{e} \rightarrow M_{e}$ to $X$, is $\Lambda$-reducible.

Proof. By Ratner's Theorem [R], there is a connected Lie group $L, \sigma(G) \subset L \subset$ $H$, such that $\lambda_{*}\left(\mu_{e}\right)$ lies on an $L$-orbit in $\Gamma \backslash H$ of the form $h \Gamma h^{-1} \cap L \backslash L$, where $h \in H$ and $h \Gamma h^{-1} \cap L$ is a lattice in $L$. In other words, there is some $h \in H$ such that $\varphi\left(X_{e}\right) \subset \Gamma h L$ (a.e.). Thus, we can write $\varphi(x)=\omega(x) h \theta(x)$ where $\omega: X_{e} \rightarrow \Gamma$ and $\theta: X_{e} \rightarrow L$ are measurable. We then have

$$
\theta(g x)^{-1} h^{-1} \omega(g x)^{-1} \alpha_{X}(g, x) \omega(x) h \theta(x)=\sigma(g) .
$$

The cocycle $\beta(g, x)=\omega(g x)^{-1} \alpha_{X}(g, x) \omega(x)$ is equivalent (as a cocycle into $\Gamma$ ) to $\alpha_{X}$ and satisfies

$$
\beta(g, x)=h \theta(g x) \sigma(g) \theta(x)^{-1} h^{-1} \in h L h^{-1} .
$$

Therefore, $\beta(G \times X) \subset \Gamma \cap h L h^{-1}$. Hence, the $G$-action on $P_{X}$ is $\Lambda$-reducible for $\Lambda=\Gamma \cap h L h^{-1}$ and $\Lambda$ is a lattice in $h L h^{-1}$.

Lemma 3.7. Let $L$ be as in Proposition 3.6. Then $L$ acts properly on $J \backslash H$. Proof. $\Gamma$ acts properly by assumption, and hence so does $\Lambda$. Since $\Lambda$ is a lattice in $\mathrm{L}$, $\mathrm{L}$ will also act properly. For convenience, we recall the proof of this latter assumption.

We first recall there is a compact symmetric set $B \subset L$ such that $L=B \Lambda B$. Namely, choose a compact symmetric $B \subset L$ whose projection [B] to $L / \Lambda$ has measure at least 3/4. Then, for any $h \in L, h([B]) \cap[B]$ has positive measure; i.e., $h B \cap B \Lambda$ is nontrivial and so $h \in B \Lambda B$. Now take any $A \subset J \backslash H$ that is compact. If $h \in L$ and $A h \cap A \neq \varnothing$, write $h=b \lambda c$ where $\lambda \in \Lambda$, and $b, c \in B$. Then $(A B) \lambda \cap A c^{-1} \neq \varnothing$, i.e., $(A B) \lambda \cap A B \neq \varnothing$.

Letting $\Lambda_{0} \subset \Lambda$ be $\{\lambda \in \Lambda \mid A B \lambda \cap A B \neq \varnothing\}$, we have $\Lambda_{0}$ is finite since $A B$ is compact, and $h \in B \Lambda_{0} B$ which is compact.

Proposition 3.8. There is a discrete subgroup $F \subset \Gamma$ with the following properties:

(i) $F$ is finitely generated.

(ii) The action of $G$ on $P_{X} \rightarrow X$ is F-reducible.

(iii) If we let $N$ be the Zariski closure of $F$, then $N$ acts properly on $J \backslash H$.

Proof. The group $[L, L] \subset H$ is algebraic. Let $\beta: G \times X \rightarrow \Lambda$ be a cocycle corresponding to the $G$-invariant $\Lambda$-reduction of $P_{X}$, and $\pi: L \rightarrow L /[L, L]$. 
Then $\pi \circ \beta$ is a cocycle taking values in the countable abelian group $\pi(\Lambda)$. It follows from [Z1, Theorem 9.1.1] (using Kazhdan's property) that $\pi \circ \beta$ is equivalent to a cocycle taking values in a finite subgroup, and hence $\beta$ is equivalent to a cocycle taking values in a subgroup $\Lambda_{1} \subset \Lambda$ such that $\Lambda_{1} \supset$ $\Lambda \cap[L, L]$ as a subgroup of finite index. It follows that $\Lambda_{1}$ is contained in an algebraic group $L_{1}$ such that $L_{1} \cap[L, L]$ is of finite index in $L_{1}$. Since $G$ is a Kazhdan group, any cocycle into a countable group is equivalent to one taking values in a finitely generated subgroup [Z8, Lemma 2.2]. Therefore, there is a finitely generated $F \subset \Lambda_{1}$ such that the $G$-action on $P_{X} \rightarrow X$ is $F$-reducible. Letting $N$ be the Zariski closure of $F$, we have $N \subset L_{1}$, and hence $N / N \cap L$ is finite. Properness of the $N$ action on $J \backslash H$ then follows from Lemma 3.7.

Now let $Q_{X} \rightarrow X$ be the principal $H$-bundle obtained by pulling back $Q_{e} \rightarrow$ $(J \backslash H)_{e}$ to $X$. Alternatively, $Q_{X}$ is the principal $H$-bundle associated to the $\Gamma$-bundle $P_{X} \rightarrow X$ and the embedding $\Gamma \rightarrow H$. Since the $G$-action on $P_{X}$ is $F$ reducible, it is clear that the algebraic hull of the $G$-action on $Q_{X}$ is contained in $N$. On the other hand, this algebraic hull is exactly $\overline{\sigma(\widetilde{G})}$ (the Zariski closure) in which $\sigma(\widetilde{G})$ is of finite index. (This assertion follows from the Borel density theorem. See, e.g., [Z4].) Thus a conjugate of $\sigma(\widetilde{G})$ is contained in $N$. We now wish to show that, in fact, some conjugate of $G$ itself is contained in $N$. (This would follow of course if we knew $\sigma$ was conjugate to the original realization $\widetilde{G} \rightarrow G \subset H$, but this is what we have not yet established.)

Lemma 3.9. $G$ is contained in a conjugate of $N$.

Proof. Since there is a $G$-invariant reduction of $P_{X} \rightarrow X$ to $F$ and $F \subset N$, the algebraic hull of the $G$-action on $Q_{X}$ is clearly contained in $N$.

Consider now the right $H$-action on $J \backslash H$. For each $y \in J \backslash H$, let $\mathfrak{h}_{y} \subset \mathfrak{h}$ be the Lie algebra of the stabilizer of $y$. Then $y \mapsto \mathfrak{h}_{y}$ defines a $\Gamma$-map $\eta: J \backslash H \rightarrow \operatorname{Gr}(\mathfrak{h})$ (in fact, an $H$-map) where $\operatorname{Gr}(\mathfrak{h})$ is the Grassmann variety of $\mathfrak{h}$. Since $G$ acts on the left of $J \backslash H$, it is straightforward to check that $\eta(g y)=\eta(y)$ for all $y \in J \backslash H$ and $g \in G$.

Write $J=R \ltimes U$ a Levi decomposition, so $U$ is the unipotent radical. Let $\mathfrak{u}$ be the Lie algebra of $U$, on which $J$ acts. We can write $o \subset \mathfrak{u}_{1} \subset$ $\mathfrak{u}_{2} \subset \cdots \subset \mathfrak{u}_{r}=\mathfrak{u}$, where $\mathfrak{u}_{i} \subset \mathfrak{u}$ is a $J$-invariant ideal and the $J$-action on $\mathfrak{u}_{i+1} / \mathfrak{u}_{i}$ is irreducible (and, in particular, is trivial on U). Since each $\mathfrak{u}_{i}$ is $J$-invariant, we can define an $H$-equivariant map $w_{i}: J \backslash H \rightarrow \operatorname{Gr}(\mathfrak{u})$ (the Grassmann variety of $\mathfrak{u})$ by $w_{i}(y)=\operatorname{Ad}(h)^{-1} \mathfrak{u}_{i}$ where $y=J h$. For any $g \in G$, let $l(g)$ be left translation on $J \backslash H$. Then $w_{j} \circ l(g)$ will also be $H$ equivariant and $\left[w_{j} \circ l(g)\right]([e])=w_{j}(g)=\operatorname{Ad}(g)^{-1} \mathfrak{u}_{j}=\mathfrak{u}_{j}($ since $\operatorname{Ad}(G)$ is trivial on $\mathfrak{u})=w_{j}([e])$. Since $w_{j} \circ l(g)$ and $w_{j}$ agree at a point and are $H$-invariant, they are equal.

Define $w: J \backslash H \rightarrow \operatorname{Gr}(\mathfrak{h}) \times \prod_{i} \operatorname{Gr}(\mathfrak{u})=V$ by $w=\left(\eta, w_{1}, \ldots, w_{r}\right)$. This is then an $H$-equivariant, $G$-invariant map. The map $y \mapsto(y, w(y))$ therefore factors to a map $s: J \backslash H / \Gamma \rightarrow(J \backslash H \times V) / \Gamma$. Since the image of $y \mapsto w(y)$ clearly lies in the orbit of $\left(\mathfrak{j}, \mathfrak{u}_{1}, \ldots, \mathfrak{u}_{r}\right)$ under $H$, the map $s$ can be viewed as a section of the bundle $\left(J \backslash H \times H / H_{0}\right) / \Gamma \rightarrow J \backslash H / \Gamma$ where $H_{0}$ is the stabilizer of $\left(\mathfrak{j}, \mathfrak{u}_{1}, \ldots, \mathfrak{u}_{r}\right)$ in $H$. It follows from [Z4] that the algebraic hull of the 
$G$-action on the principal $H$-bundle $(J \backslash H \times H) / \Gamma \rightarrow J \backslash H / \Gamma$ is contained in $H_{0}$.

It follows that the same is true for the algebraic hull of the $G$-action on the pullback of this bundle to $X$, which is clearly isomorphic to $Q_{X}$. As we have already observed, this algebraic hull is contained in $N$. We clearly have that $H_{0}=N_{H}(\mathfrak{j}) \cap \bigcap_{i} N_{H}\left(\mathfrak{u}_{i}\right)$. It follows that for some $h \in H$ and some conjugate $G_{1}$ of $\sigma(\widetilde{G})$ that

$$
G_{1} \subset h N h^{-1} \cap N_{H}(\mathfrak{j}) \cap \bigcap_{i} N_{H}\left(\mathfrak{u}_{i}\right) .
$$

By Proposition 3.8, $N$, and hence any conjugate of $N$, acts properly on $J \backslash H$. Therefore, $G_{1}$ acts properly on the right of $J \backslash H$, so the intersection of any conjugate of $G_{1}$ with $J$ is compact. Since $G_{1}$ is semisimple with no compact factors and $G_{1}$ normalizes $j$, we have $\mathfrak{g}_{1} \cap \mathbf{j}=(0)$.

Now consider the subgroup $G_{1} J=G_{1} R U$. Note that each $\mathfrak{u}_{j}$ is an ideal, i.e. is invariant under all $G_{1} J$. Since $G_{1}$ and $R$ are reductive, $G_{1}$ normalizes $R U$, and $\mathfrak{g}_{1} \cap \mathfrak{j}=(0)$, in the quotient group $G_{1} R U / U$ we can conjugate $G_{1} U / U$ so that it centralizes $R U / U$. (We are using here the fact that there are no nontrivial local homomorphisms from $G$ to $J$.) Choosing a lift of this semisimple group in $G_{1} R U$, we deduce the following: there is a conjugate $G_{2}$ of $G_{1}$ in $G_{1} R U$ such that $G_{2}$ centralizes $R$. Furthermore, $G_{2} R$ acts on each $\mathfrak{u}_{i+1} / \mathfrak{u}_{i}$, and the representation of $R$ on this space is irreducible. It follows that $G_{2}$ acts by scalars on $\mathfrak{u}_{i+1} / \mathfrak{u}_{i}$ and, hence, that $G_{2}$ acts on $\mathfrak{u}$ by a solvable group. Since $G_{2}$ is simple, $G_{2}$ acts trivially on $\mathfrak{u}$. Thus $G_{2}$ commutes with both $R$ and $U$, and hence $G_{2} \subset Z_{H}(j)$. This shows that some conjugate of $\sigma(\widetilde{G})$ is contained in $h N h^{-1} \cap Z_{H}(J)$. But by remarks at the beginning of this section, since any representation $\widetilde{G} \rightarrow Z_{H}(J)$ has image equal to a conjugate of $G$ in $Z_{H}(J)$, it follows that for some (possibly different) $h, h N h^{-1} \cap Z_{H}(J) \supset G$. This completes the proof.

Lemma 3.10. There is a finitely generated discrete group $F \subset \Gamma$ such that:

(a) There is a G-invariant subset of finite (smooth) measure in $J \backslash H / F$;

(b) If $N$ is the Zariski closure $F$, then $N$ acts properly on $J \backslash H$; and

(c) $G$ is contained in $N$.

Proof. Consider the diagram

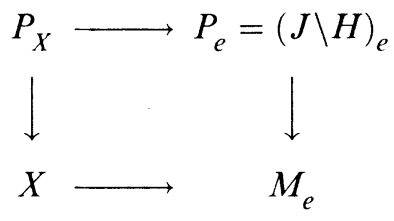

This yields a diagram

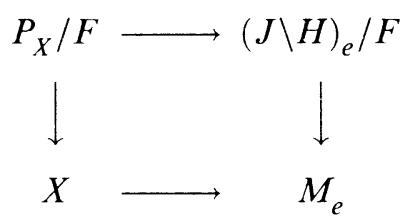


By Proposition 3.8, there is a $G$-invariant section of $P_{X} / F \rightarrow X$ and, hence, a finite $G$-invariant measure $\theta_{e}$ in $P_{X} / F$ that projects to the given measure on $X$. Pushing this measure forward to $(J \backslash H)_{e} / F$, we obtain a finite $G$ invariant measure on this space that projects to $\mu_{e}$. This is all still within one ergodic component. The space of finitely generated subgroups of $\Gamma$ is countable. Therefore, there is a set of ergodic components of positive $\nu$-measure, say $E_{0}$, on which the assignment $e \mapsto F$ will be constant. The measure $\theta=\int_{e \in E_{0}}^{\oplus} \theta_{e}$ is a finite $G$-invariant measure on $J \backslash H / F$ that projects to a set of positive measure in $M$ (namely, the union of the ergodic components $e \in E_{0}$ ). Since the bundle $J \backslash H / F \rightarrow M=J \backslash H / \Gamma$ has discrete fibers, $\theta$ is the restriction of the smooth measure on $J \backslash H / F$ to a set of positive measure. The remaining assertions of Lemma 3.10 now follow from Proposition 3.8, Lemma 3.9, and a conjugation.

Lemma 3.11. G fixes every point of $J \backslash H / N$.

Proof. By Rosenlicht's theorem [Ro], there is a Zariski open ( $\mathbb{C}$-connected) $J \times G$-invariant subset $U$ of $H / N$ such that $J \backslash U$ is a variety on which $G$ acts algebraically. Pushing the measure of Lemma 3.10(a) forward under the map $J \backslash H / F \rightarrow J \backslash H / N$, we deduce that there is a finite $G$-invariant measure on $J \backslash U$ that is the restriction of a smooth measure to a subset of positive measure. Since the $G$-action on $J \backslash U$ is algebraic, the Borel density theorem implies any finite invariant measure is supported on $G$-fixed points. Therefore, we deduce that there is a set of $G$-fixed points in $J \backslash U$ of positive smooth measure, and, since the action is algebraic, $G$ pointwise fixes $J \backslash U$. This means there is a dense set of $G$-fixed points in $J \backslash H / N$. However, since the $N$ action on $J \backslash H$ is proper, $J \backslash H / N$ is Hausdorff, and hence $G$ fixes all points of $J \backslash H / N$.

Completion of Proof of Theorem. Choose a 1-parameter $\mathbb{R}$-split subgroup $a(t)$ in $G$. Since the root spaces for $a(t)$ in $\mathfrak{h}$ corresponding to nonzero roots generate a normal subgroup of $H$, it suffices to see that all these root spaces are in $\mathfrak{n}$. If $\mathfrak{u} \subset \mathfrak{h}$ is such a 1 -dimensional eigenspace and $\mathfrak{u} \subset \mathfrak{j}+\mathfrak{n}$, then we must have $\mathfrak{u} \subset \mathfrak{n}$ since $G$ (and in particular $a(t)$ ) centralizes $\mathfrak{j}$. Therefore, we need only consider $\mathfrak{u} \not \subset \mathfrak{j}+\mathfrak{n}$. Let $U(s) \subset H$ be the corresponding 1-parameter subgroup. Then

$$
\begin{aligned}
a(t) U(s) N & =a(t) U(s) a(t)^{-1} N \\
& =U\left(e^{\lambda t} s\right) N \quad(\text { since } a(t) \in G \subset N)
\end{aligned}
$$

for some $\lambda \in \mathbb{R}, \lambda \neq 0$ This means that $U^{+}=\{U(s) N \mid s>0\}$ is contained in a single $G$-orbit in $H / N$. However, since $G$ acts trvially on $J \backslash H / N$, it follows that $U^{+}$is contained in a single $J$-orbit. By Lemma 3.10, $N$ acts properly on $J \backslash H$, so $J \backslash H / N$ is Hausdorff and the $J$-orbits in $H / N$ are closed. Therefore, $U^{+}$is contained in the $J$-orbit of $e N$, i.e. in $J N \subset H$. However, $J N$ is a closed submanifold whose tangent space at $e \in H$ is $\mathfrak{j}+\mathfrak{n}$, and, since $u \not \subset j+\mathfrak{n}$, we have $U(s) \notin J N$ for $s>0$ but sufficiently small. This contradiction shows all such $\mathfrak{u}$ are in $\mathfrak{n}$ and, hence, that $\mathfrak{n}=\mathfrak{h}, N=H$, and $J$ is compact (by Lemma 3.10). 


\section{REFERENCES}

[BL] Y. Benoist and F. Labourie, Sur les espaces homogènes modelés de variétés compactes, preprint.

[B] A. Borel, Compact Clifford-Klein forms of symmetric spaces, Topology 2.(1963), 111-122.

[CM] E. Calabi and L. Markus, Relativistic space forms, Ann. of Math. (2) 75 (1962), 63-76.

[CZ] K. Corlette and R. J. Zimmer, Superrigidity for cocycles and hyperbolic geometry, preprint.

[G] W. Goldman, Projective geometry on manifolds, Univ. of Maryland Lecture Notes, 1988.

[K] T. Kobayashi, Proper action on a homogeneous space of reductive type, Math. Ann. 285 (1989), 249-263.

[KO] T. Kobayashi and K. Ono, Note on Hirzebruch's proportionality principle, J. Fac. Sci. Univ. Tokyo Sect. IA Math. 37 (1990), 71-87.

[Ku] R. S. Kulkarni, Proper actions and pseudo-Riemannian space forms, Adv. Math 40 (1981), $10-51$.

[R] M. Ratner, On Raghunathan's measure conjecture, Ann. of Math. (2) 134 (1991), 545-607.

[Ro] M. Rosenlicht, A remark on quotient spaces, An. Acad. Brasil Ciênc. 35 (1963), 487-489.

[W] J. A. Wolf, The Clifford-Klein space forms of indefinite metric, Ann. of Math. (2) 75 (1962), 77-80.

[Z1] R. J. Zimmer, Ergodic theory and semisimple groups, Birkhäuser, Boston, MA, 1984.

[Z2] _ Strong rigidity for ergodic actions of semisimple Lie groups, Ann. of Math (2) 112 (1980), 511-529.

[Z3] _ On the algebraic hull of the automorphism group of a principal bundle, Comment. Math. Helv. 65 (1990), 375-387.

[Z4] __ Ergodic theory and the automorphism group of a G-structure, Group Representations, Ergodic Theory, Operator Algebras, and Mathematical Physics (C. C. Moore, ed.), Springer Verlag, New York, 1987.

[Z5] _ Superrigidity, Ratner's theorem, and fundamental groups, Israel J. Math. 74 (1991), 199-207.

[Z6] _ Extensions of ergodic groups actions, Illinois J. Math. 20 (1976), 373-409.

[Z7] _ Kazhdan groups acting on compact manifolds, Invent. Math. 75 (1984), 425-436.

[Z8] _ Groups generating transversals to semisimple Lie group actions, Israel J. Math. 73 (1991), 151-159.

[Z9] _ Topological superrigidity, preprint.

Department of Mathematics, University of Chicago, 5734 University Avenue, ChicAGo, Illinois 60637

E-mail address: zimmer@math.uchicago.edu 